\title{
INVESTIGATION OF
}

\section{PROLONGED PYREXIA}

\author{
By T. W. Preston, M.D.(LOND.), M.R.C.P.(LOND.) \\ Physician, Croydon General Hospital; Hon. Lieut.-Col. R.A.M.C., lately Officer i/c of a Medical Division \\ India Command
}

\section{Preliminary Considerations}

By prolonged pyrexia will be understood pyrexia of over ten days' duration. For the purposes of this article it will be assumed that a careful clinical examination has failed to reveal physical signs of a definite character.

Confronted with such a case it will rarely be possible to make an immediate diagnosis and investigation resolves itself into carrying out certain routine tests which will reduce a large number of "possibles" into a lesser number of "probables" from which the final diagnosis can finally be determined by some specific test. The late Sir Raymond Crawford was fond of saying to his students, "Rare things are rare and common things are common," and this is an adage which may profitably be borne in mind in this type of case.

At this stage, the following investigations should be carried out as routine.

(I) Leucocyte and differential leucocyte count.A high polymorphoneucleocytosis will make it extremely probable that the patient is suffering from some pyogenic infection-very probably a hidden collection of pus, and further investigations should be concentrated in this direction. A leucopenia, especially with a relative lymphocytosis, will indicate an entirely different field for further inquiry, whilst large numbers of monocytes or eosinophiles will definitely narrow the field. Where the picture is indefinite, repeated counts at three-day intervals should be made. It must be remembered that the picture may vary from day to day and, moreover, the picture may be misleading if the patient has been having a course of sulphonamide.

(2) Blood culture.-One sample of blood should be cultured on glucose broth, a second on a medium, suitable for the isolation of the Enteric Group (e.g. McConkey's medium). A positive culture will usually be conclusive but a negative result excludes nothing.

(3) Agglutination reactions against B. typhosus and B. paratyphosis A. and B.; M. melitensis and
Br. abortus of Bang. A single reading against theenteric group will frequently be inconclusives (especially if the patient has been innoculated), buto a series of three tests at two-day intervals may show a rising titre of diagnostic significance.

(4) $A$ blood film should be examined for malarial N parasites if the patient has lived in a tropical ors sub-tropical country; if necessary this should be repeated several times, if possible during a rigor.

Pending the result of these investigations, theo case will be carefully reviewed, a study made $\mathrm{Bf}_{-}$ the type of fever as shown by a four-hourly chad, $\vec{\square}$ and inquiry made into the patient's recent and remote movements. This is particularly important ${ }_{3}^{\mathbb{\Phi}}$ at the present time when so many men and womeng have recently returned from military service overseas. In considering residence overseas it gुsco desirable to ascertain with some accuracy theo particular location and in obscure cases to consulft the relevant authorities as to the diseases endemic and epidemic. A general statement that the patient has been "in Africa" or "in India" mayo lead those unfamiliar with these countries to wasteळ time looking for diseases which in fact never occur $\stackrel{\square}{\Rightarrow}$ in the areas concerned.

An ex-soldier was suffering from an obscure pyrexia. Information that he had served in India led the doctor in charge to institute investigations for Kala-azar. More detailed inquiry showed that in India he had been stationed 3 exclusively in Hyderabad state and Bombay Presidencyareas in which Kala-azar is as little prevalent as in the $\frac{\sigma}{3}$ Isle of Wight.

After these preliminary considerations, the various diseases likely to be the cause of prolonged pyrexiao may be enumerated and briefly reviewed. They may conveniently be subdivided into the following groups:

(a) Diseases of World-Wide Incidence.

i. Likely to be associated with a polymor-N phonucleo-leucocytosis.

ii. Likely to be associated with a leucopenia.

iii. Likely to be associated with an indefinite bloodo picture. 
(b) Diseases peculiar to certain areas.

(a) (i) Diseases of World-Wide Incidence Associated with Polymorphonucleoleucocytosis.

i. Septicaemia (including Bacterial Endocarditis).

ii. Pyaemia.

iii. Hidden collection of pus.-It is neither practicable nor desirable to discuss the numerous conditions included under this heading, but special mention may be made of perinephric abscess and "carbuncle of the kidney." There are probably few clinicians of experience who have not at some time had under their care cases of obscure pyrexia which have long eluded diagnosis and which have ultimately turned out to have had a perinephric abscess. In view of the obscure signs, special attention should always be concentrated on the exclusion of this most difficult condition. A previous history of boils will lead to a suspicion of the closely allied condition of "carbuncle of the Kidney." (En passant, a warning may be uttered at the too facile dismissal of a staphylococcus grown from blood or urine culture as a contamination. Mention may be made of a case ultimately diagnosed as carbuncle of the kidney which had remained undiagnosed for many weeks. Perusal of the case papers revealed that early after admission a urine culture had grown a staphylococcus -and disregarded.)

\section{(a) (ii) Diseases of World-Wide Incidence Associated with Leucopenia.}

I. Enteric Group of Fevers. - It is essential to exclude this group in any case of prolonged pyrexia. Characteristically.the temperature chart will show a step-like rise and a relatively slow pulse, but all types of fever may occur. Although commonest in tropical countries, especially when associated with faulty hygiene, it occurs sporadically and in minor epidemics in this country.

The surest method of diagnosis is by blood culture. This is most likely to be positive in the early stages but is well worth carrying out at any stage.

Failing a positive blood culture, isolation of the bacilli from the stools is successfully undertaken in many cases when an efficient technique is employed; although most likely to give positive results after the third week, an increasing number of bacteriologists are now successful in the early stages.

\section{Agglutination Reactions:}

" $H$ " agglutination is indicative of infection with the homologous organism in an uninnoculated person. If the patient has been previously inno culated however (even twenty years previously $\mathrm{H}$-agglutination is valueless.

" $O$ " agglutination is not specific for separatez members of the " $E$ " group but is not produced by innoculation. Hence, a rising titre in serial tests. is of diagnostic significance of enteric infection $\overrightarrow{\overrightarrow{\vec{S}}}$ but is not specific.

" $V$ " agglutinins are highly specific.

It is suggested that the terms "+ Widal" and " - Widal" should be abandoned as valueless and misleading; the only report of clinical value is a rising titre in at least two and preferably three tests done at two-day intervals.

2. Undulant Fever (Abortus type) is seen occa sionally in all parts of the world. Clinically it is milder than Melitensis infections (see under (B)); i has few characteristic features of diagnostic signio ficance and it is rarely diagnosed until it has run? for many weeks and numerous investigations made with negative result; if the pyrexia assumesu the characteristic undulant type the case may bet suspected clinically; but frequently the fever is continuous or irregular. Diagnosis is establishedr by agglutination of $\mathrm{I}-\mathrm{roO}$ or more to $\mathrm{Br}$. Abortwos of Bang. Infection is due to ingestion of infectêd milk or contiguity to infected animals, but often $\frac{3}{10}$ is impossible to trace any connection and the clinical history may be most misleading.

A soldier was admitted to a Military Hospital in Scotla욤 with the following history: Six weeks previously whistet stationed in the English Midlands he had been admitted to hospital with acute tonsillitis: contrary to establisfed practice his tonsils had been enucleated during the stage of acute infection. After a stormy convalescence he wass sent on leave to his home in Scotland where he spent most of the time in bed feeling unwell and running irregulan fever. At the expiration of his leave he consulted a locak doctor who sent him to the nearest Military Hospital as a을 "P.U.O." Physical examination was negative and although blood culture was sterile a provisional diagnosiso of streptococcal septicaemia seemed, under the circum-3 stances, not unreasonable. This diagnosis seemed strengthened when the temperature subsided coincidentally witho the exhibition of sulphonamide. After a few days. however, the temperature rose again, and the diagnosis was only established several weeks later when agglutina tion to $\mathrm{Br}$. Abortus was obtained in high dilution.

3. Agranulocytosis.-This condition is to be borne in mind especially in view of the prevalent, and sometimes injudicious, use of the suphonamideo group of drugs. Differential diagnosis may be difficult from aplastic anaemia, acute aleukaemico leukaemia and acute mononucleosis. Examination of sternal marrow by a skilled haematologist mayo help.

(A) (iii) Diseases of World-Wide Incidence with Indefinite Blood Picture.

I. Atypical Pneumonia.-Usually the fever sub- 
sides within ro days, but in more prolonged cases the absence of physical signs will make diagnosis difficult. There may be an increase of monocytes. The diagnosis can only be established by radiography.

2. Influenza.-Except in epidemics, this diagnosis should never be made until other conditions have been excluded; the diagnosis should only be provisional so long as fever persists and investigations must be continued. There may be leucopenia and the pulse relatively slow, but these findings are inconstant. If fever persists for more than two weeks it is wise to assume either that the diagnosis is wrong or that there are complications (especially pneumonia or empyaema).

3. Acute Miliary Tuberculosis.

4. Relapsing Fever.-Although commonest in the East, it occurs in Europe and is reported from time to time in Ireland (louse-borne). (The tick-borne occurs in Central Africa.) It is characterised by five to seven days of high fever followed by a crisis and ten to fourteen days remission followed by relapse. This may be repeated once or several times. Diagnosis is established by detection of the spirochaete in thick blood films taken during fever.

5. Rat-Bite Fever produces a relapsing type of fever difficult to distinguish from relapsing fever. There may be a polymorphonucleocytosis. The history, local lymphangitis and a raised erythematous rash will direct the diagnosis. The causative spirillum may be found in the blood but its isolation is difficult.

6. Typhus Fevers.-The true louse-borne or epidemic typhus is world-wide. Tick-borne, miteborne and flea-borne varieties occur endemically, various sub-types in various districts. In endemic areas and in epidemics diagnosis on clinical grounds is easy, otherwise diagnosis is established by the Weil-Felix reaction. (Note: sporadic cases are unlikely.)

7. Glandular Fever.-The common glandular types and the rare Anginose type do not call for description in a discussion on Prolonged Pyrexia. The febrile type, however, may present extreme difficulties in diagnosis. Adenitis may be transitory and too slight to be significant, and clinically the course and symptoms may be uncharacteristic. Pyrexia may be prolonged with recurrences and apyrexial periods. Undulant fevers, lymphadenoma and leukaemia may be suspected. Although monocucleosis is characteristic (and probably always occurs at some stage), the blood picture is extremely variable and may change from day to day. There may be leucopenia or leucocytosis and polymorphs, lymphocytes or monocytes may predominate. In suspected cases repeated blood examinations should be made. A count of over
30,000 per c.mm. is unusual and raises suspicion of a leukaemia. A positive Paul-Bunnell reaction ib a titre of $I-64$ is diagnostic. (There is no doubto that this group calls for further clarification.)

8. Lymphadenoma may be associated with pyrexiag of a relapsing type (Pel-Ebstein) or irregular. The blood picture is not characteristic and diag nosis can only be established by biopsy of a glandঙ्ञ Great difficulty in diagnosis may occur when the mediastinal or mesenteric glands are enlarged witho no appreciable superficial adenitis.

9. Leukaemias.-Atypical leukaemias witl居 pyrexia and aleukaemia may present difficulty® Diagnosis depends on repeated blood examinations. and examination of sternal marrow by a skilled haematologist.

\section{(B) Diseases Peculiar to Certain Areas.}

I. Malaria. With a history of relapse in tropical or sub-tropical country it is always necessary to exclude malaria. The followings practical points should be borne in mind:

Duration of liability to relapse: M.T., rare afteff्f eighteen months; B.T., common up to two years and may be up to three or four years; Quartaf? very persistent-four years or more.

Well-authenticated cases have occurred $\overparen{D}$ apparent first attacks in this country in persoms who have not had clinical malaria overseas.

One negative blood film does not exclugle Malaria; it may be necessary to examine magyo films before parasites are found-preferably takent? during a rigor.

A soldier was admitted to hospital in India with per sistent pyrexia. Six blood films were taken and examined by an experienced pathologist with negative results. seventh film was literally swarming with M.T. parasites.

Occasionally parasites (especially M.T.) may be를 discovered on sternal puncture which have eluded detection in blood films.

It is emphasised that the characteristic regular? intermittent fever may be replaced by a fevero irregular or continuous.

Occasionally it is justifiable to try the therapeutic effect of Quinine in spite of negative films.

2. Kala-azar (Leishmaniasis).-Although fairly? wide-spread this condition has a somewhat patchy distribution and is practically unknown in some areas in close proximity to others where it is common. In Africa it is mainly found on the East coast, in Sudan and the Blue Nile, Ethiopia $\mathcal{O}^{\circ}$ and on the Mediterranean coast; in Europe, on the south coast of Spain, Malta and Crete, Sicily and the "toe" of Italy. In India-a few caseso occur in Madras - then passing north the country 
is free of it until we come to the Ganges delta; it is common in Bengal and Assam, but is never met west of Lucknow, Western India being free. It occurs in parts of China, Manchukuo, Mongolia, Transcaucasia, and Russian Turkestan, and in parts of South America.

The fever is characteristically remittent, dropping in the morning, rising in the early afternoon, falling again towards evening, and rising again during the night. This will be shown by keeping a threehourly chart. This may continue for many weeks followed by a period of partial apyrexia followed by a relapse. This may continue for many months.

Absence of malaise and symptoms may be striking. It is a clinical experience never to be forgotten to visit a hospital where cases of Kalaazar are being treated; to inspect first the temperature charts showing fever to $103^{\circ}$ and $104^{\circ}$, and then to see the patients sitting up in bed eating a hearty meal and demanding to be allowed up! It is frequently difficult to persuade Indian and African patients to remain in hospital and often impossible to confine them to bed.

Leucopenia is a striking and constant featureusually below 3,000 per c.mm. and often down to I,ooo per c.mm., the deficiency being mainly in polymorphs.

Enlargement of the spleen occurs approximately at the same rate as the pregnant uterus in the opposite direction. Thus in early cases the spleen will be just palpable, in a case of six months duration it may be expected in the region of the umbilicus.

Diagnosis can only be established by demonstrating L-D bodies in material obtained by sternal or splenic puncture. Although less reliable, sternal puncture should always be tried first on account of its greater safety and the material cultured as well as examined in direct films. Splenic puncture should be performed by a rapid thrust with a fine-bore needle; the drop of fluid thus obtained is quite sufficient for examination. Prolonged aspiration through a wide-bore needle is unnecessary and dangerous. It is emphasised that recognition of $\mathrm{L}-\mathrm{D}$ bodies requires the familiarity which is only gained by experience. It is futile for the clinician to subject his patient to the inconvenience (and possible danger) of sternal or splenic puncture unless he has arranged for the material to be examined by a pathologise experienced in Kala-azar.

Napier's Aldchyde test is valuabre and easily carried out, but it rarely gives a positive result until the disease is of three to four months duration $\bar{\square}$ It is therefore useless for early diagnosis, but a. negative reaction in a case of long-standing illnes can be safely taken to exclude Kala-azar. Doubt ful positive reactions are stated to occur in certaing other diseases (advanced tuberculosis, and leprosy)음

3. Undulant Fever (Melitensis type) is wide $\frac{\bar{s}}{\square}$ spread in goat-rearing districts of the tropics and sub-tropics. The undulant character of the feven is more constant than in the Abortus type and? usually the patient is more ill. Sweating may be profuse and there is complaint of headache, $\overrightarrow{\vec{q}}$ picture which may be mistaken for acute rheus matism. The spleen may be enlarged. The pulso is relatively slow. There is usually leucopenia. with relative lymphocytosis. Agglutination tod I-IOo or over to M. Melitensis establishes the diagnosis.

A soldier was invalided home from North Africa as case of Pulmonary Tuberculosis. His prolonged fevef seemed inconsistent with the apparently healed apf inactive tuberculous focus seen radiologically. Agglutiga $=$ tion reactions gave a strong positive to $M$. Melitensis.

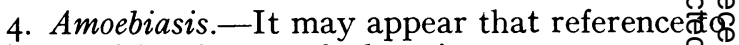
this condition is out of place in a paper on p:0 longed pyrexia since localising symptoms aี่ signs might reasonably be anticipated. It ôis. emphasised, therefore, that Amoebic Dysentery occasionally an insidious disease and bopep symptoms may occur ten years or longer aftef original infection (which may never have beers diagnosed). When, therefore, obscure pyrexiक occurs in persons with a past record of tropica\$ residence, examination of the stools and carefu\$ palpation of the liver is indicated. Sometimes liver abscess may occur in an impalpable live and only recognised by "humping" of the diaכ phragm revealed by screening. (Note: such 变 liver, however, would usually be painful on pressure over the intercostal spaces.) It may be noted that whilst a leucocytosis (with increased polymorphsक is usual it may be only of moderate degree and may be absent. In Endemic areas the therapeutig effect of emetine is sometimes regarded as a usefu and justifiable test. 